\title{
Efficacy of Dupilumab in Atopic Dermatitis: The Patient's Perspective
}

\author{
Marjolein de Bruin-Weller (D) · Joseph F. Merola (D) - Chih-ho Hong (D) • \\ Esther Serra Baldrich (D) - Karel Ettler (D) · Debra Sierka • \\ Dimittri Delevry (D) - Zhen Chen · Ana B. Rossi
}

Received: September 2, 2021 / Accepted: September 25, 2021 / Published online: November 12, 2021

(c) The Author(s) 2021

\begin{abstract}
Introduction: Atopic dermatitis (AD), a predominantly type 2 inflammatory skin disease, affects approximately $2-5 \%$ of adults, with a high burden of disease. In moderate-to-severe $\mathrm{AD}$, lesions can be extensive and pruritus intense with patients experiencing skin pain, sleep and mental health disturbances, and diminished quality of life (QoL).

Methods: The objective of this study was to evaluate the efficacy of dupilumab for the treatment of $\mathrm{AD}$ from the patients' perspective using patient-reported outcome data from four clinical trials (CHRONOS, SOLO $1 \& 2$, and CAFÉ) in
\end{abstract}

Electronic Supplementary Material The online version of this article (https://doi.org/10.1007/s13555021-00621-w) contains supplementary material, which is available to authorized users.

M. de Bruin-Weller

Department of Dermatology and Allergology,

National Expertise Center of Atopic Dermatitis,

University Medical Center, Utrecht, The Netherlands

J. F. Merola

Brigham and Women's Hospital, Harvard Medical

School, Boston, MA, USA

C. Hong

Department of Dermatology and Skin Science, University of British Columbia, Surrey, BC, Canada

C. Hong

Probity Medical Research, Waterloo, ON, Canada patients $(N=1553)$ receiving either the approved $300 \mathrm{mg}$ q2w dupilumab with/without topical corticosteroids (TCS) dose or control (placebo or placebo + TCS). Patient Global Assessment of Disease Status (PGADS) was used to measure patients' well-being and Patient Global Assessment of Treatment Effect (PGATE) was used to measure treatment efficacy. Patients were asked "Considering all the ways in which your eczema affects you, indicate how well you are doing" to assess their perception of well-being and "How would you rate the way your eczema responded to the study medication?" to assess their perception of treatment effect. Possible responses for both metrics included poor, fair, good, very good, and excellent.

Results: In all four studies, a significantly higher proportion of dupilumab-treated patients reported "Good"/"Very Good"/"Excellent" disease status from week 2 through study

\section{E. S. Baldrich}

Hospital Sant Pau, Universitat Autonoma Barcelona, Barcelona, Spain

\section{K. Ettler}

University Hospital Hradec Kralove, Hradec Kralove, Czech Republic

D. Sierka · A. B. Rossi (ه)

Sanofi Genzyme, Cambridge, MA, USA

e-mail: Ana.Rossi@sanofi.com

D. Delevry $\cdot$ Z. Chen

Regeneron Pharmaceuticals, Inc., Tarrytown, NY, USA 
end versus control (CHRONOS, 52 weeks: 69.8\% vs. $25.1 \%$; SOLO $1 \& 2$, 16 weeks: $59.5 \%$ vs. $24.6 \%$; CAFÉ, 16 weeks: $84.1 \%$ vs. $45.4 \%$; all $P<0.0001$ ), and significantly more dupilumabtreated patients reported "Good"/"Very Good"/ "Excellent" treatment efficacy versus control (CHRONOS: $72.6 \%$ vs. $24.8 \%$; SOLO $1 \& 2$ : $65.0 \%$ vs. $21.1 \%$; CAFÉ, 16 weeks: $85.0 \%$ vs. 36.1\%; all $P<0.0001)$.

Conclusion: Adult patients with $\mathrm{AD}$ perceived that dupilumab with/without concomitant TCS was highly efficacious and improved overall disease status and well-being as early as week 2 and throughout treatment periods up to 1 year.

Keywords: Atopic dermatitis; Dupilumab; Patient perception; Patient-reported outcomes; Treatment efficacy

\section{Key Summary Points}

\section{Why carry out this study?}

Recent guidelines for the treatment of atopic dermatitis (AD) established that the patient's assessment should be taken into account in defining treatment response; however, some studies have shown discord between the physician's and the patient's assessment of disease.

\section{What did the study ask?}

How do patients perceive the efficacy of dupilumab for the treatment of $\mathrm{AD}$ ?

What were the study outcomes/conclusions?

Adult patients with AD perceived that dupilumab, with or without concomitant topical corticosteroid treatment, was highly efficacious and improved overall disease status and well-being as early as week 2 and throughout treatment periods up to 1 year.

\section{What has been learned from the study?}

Overall, these study results indicate that most adult patients with AD perceive that dupilumab treatment is effective and improves well-being.

\section{DIGITAL FEATURES}

This article is published with digital features, including a video abstract, to facilitate understanding of the article. To view digital features for this article go to https://doi.org/10.6084/ m9.figshare.16677529.

\section{INTRODUCTION}

Atopic dermatitis (AD), a predominantly type 2 inflammatory skin disease characterized by pruritus (itch) and eczematous lesions, affects approximately $2-5 \%$ of adults $[1,2]$. In moderate-to-severe $\mathrm{AD}$, lesions can be extensive with intense pruritus and patients suffer with skin pain, sleep and mental health disturbances, and diminished quality of life (QoL) [3-5]. As a result of the chronic and relapsing nature of moderate-to-severe $\mathrm{AD}$, patients often require long-term, ongoing systemic treatment.

Dupilumab, a fully human monoclonal antibody, specifically binds to interleukin (IL)-4 receptor alpha and inhibits signalling of IL-4 and IL-13, two important type 2 inflammatory cytokines $[1,6]$. In clinical trials, dupilumab consistently improved clinical signs and symptoms of $\mathrm{AD}$ versus placebo, had an acceptable safety profile, and was shown to improve measures of sleep and QoL [7-15]. On the basis of the results of these trials, dupilumab is approved in the USA for adult and pediatric patients aged 6 years and above with moderateto-severe $\mathrm{AD}$ not adequately controlled with topical therapies or when those therapies are not advisable [16]. It is also approved in Europe for the treatment of moderate-to-severe $\mathrm{AD}$ in patients 12 years and older, and severe $\mathrm{AD}$ in children 6-11 years old who are candidates for systemic therapy $[17,18]$.

Patients' perceptions of their treatment benefit are becoming increasingly important in the benefit/risk assessment of therapeutic agents. A recently published international treatto-target consensus to guide the use of systemic treatment in adults with moderate-to-severe AD [19] established the patient's assessment of $\mathrm{AD}$ as an essential component in defining treatment response. The need to include the patient 
perspective when making treatment decisions was also highlighted by a study suggesting a potential discord between physician-assessed and patient-reported levels of AD disease severity and burden $[20,21]$. The objective of the current study was to evaluate the efficacy of dupilumab for the treatment of $\mathrm{AD}$ from the patient perspective using patient-reported outcome data from four randomized double-blind placebo-controlled clinical trials.

\section{METHODS}

This was a combined analysis of patient-reported treatment efficacy and disease-improvement data from four phase 3 randomized, placebo-controlled trials of dupilumab in adult patients with AD (CHRONOS [10], LIBERTY AD SOLO 1 [9], SOLO 2 [9], and CAFÉ [13]). Detailed methods for all studies, including full inclusion and exclusion criteria, have been previously reported. Briefly, patients from all studies were aged 18 years and older, with moderate-to-severe AD (Investigators Global Assessment score 3 or 4) inadequately controlled by topical corticosteroids (TCS) or cyclosporin A (CAFÉ only). Patients received dupilumab $300 \mathrm{mg}$ or placebo once weekly (qw) or once every 2 weeks (q2w) and were followed up for 16 (SOLO and CAFÉ) or 52 (CHRONOS) weeks. Patients in the CHRONOS and CAFÉ studies also received concomitant TCS. Study design differences between CHRONOS (52 weeks), SOLO 1, SOLO 2, and CAFÉ (all 16 weeks) did not allow for pooling of data; however, as SOLO 1 and SOLO 2 were of identical design, data from these two studies were pooled (SOLO 1\&2). All trials were approved by respective institutional review boards and conducted in accordance with the ethical principles outlined in the Declaration of Helsinki, the International Conference on Harmonisation Good Clinical Practice guidelines, and applicable regulatory requirements. All patients or carers provided written informed consent before participating in the trial.

Patients' assessments of treatment efficacy and disease improvement for both dupilumab monotherapy (SOLO) and concomitant TCS therapy (CHRONOS and CAFÉ) were included in the analysis, using two widely used, but unvalidated, metrics: Patient Global Assessment of Disease Status (PGADS) measures static disease severity and Patient Global Assessment of Treatment Effect (PGATE) measures dynamic treatment efficacy. For PGADS, patients rated their overall well-being based on a 5-point Likert scale from "Poor" to "Excellent". Patients were asked: "Considering all the ways in which your eczema affects you, indicate how well you are doing." Response choices were "Poor" (1), "Fair" (2), "Good" (3)," "Very Good" (4), and "Excellent" (5). For PGATE, patients rated their opinion and perceived treatment effect. Patients were asked "How would you rate the way your eczema responded to the study medication?" Ratings were on a 5-point Likert scale of "Poor" (1), "Fair" (2), "Good" (3), "Very good" (4), or "Excellent" (5).

Study outcomes included the proportion of patients achieving a good/very good/excellent response in PGADS and PGATE over time and at weeks 16 and 52.

The study population was the full analysis set (FAS) which included all randomized patients. Only dosing regimens from the approved $300 \mathrm{mg}$ q2w treatment and control (placebo or placebo + TCS) groups are reported.

\section{RESULTS}

\section{Patients}

For the 1553 patients included in this combined analysis, baseline demographics and disease characteristics were balanced between treatment groups and across studies (Table 1). Patients had a median age of 30-40.5 years and had chronic AD (median duration of disease 25.5-29 years; Table 1). Approximately half of patients across all of the studies had severe AD according to Investigator's Global Assessment ([IGA] patients with IGA 4, 46.7-50.0\%; Table 1). 


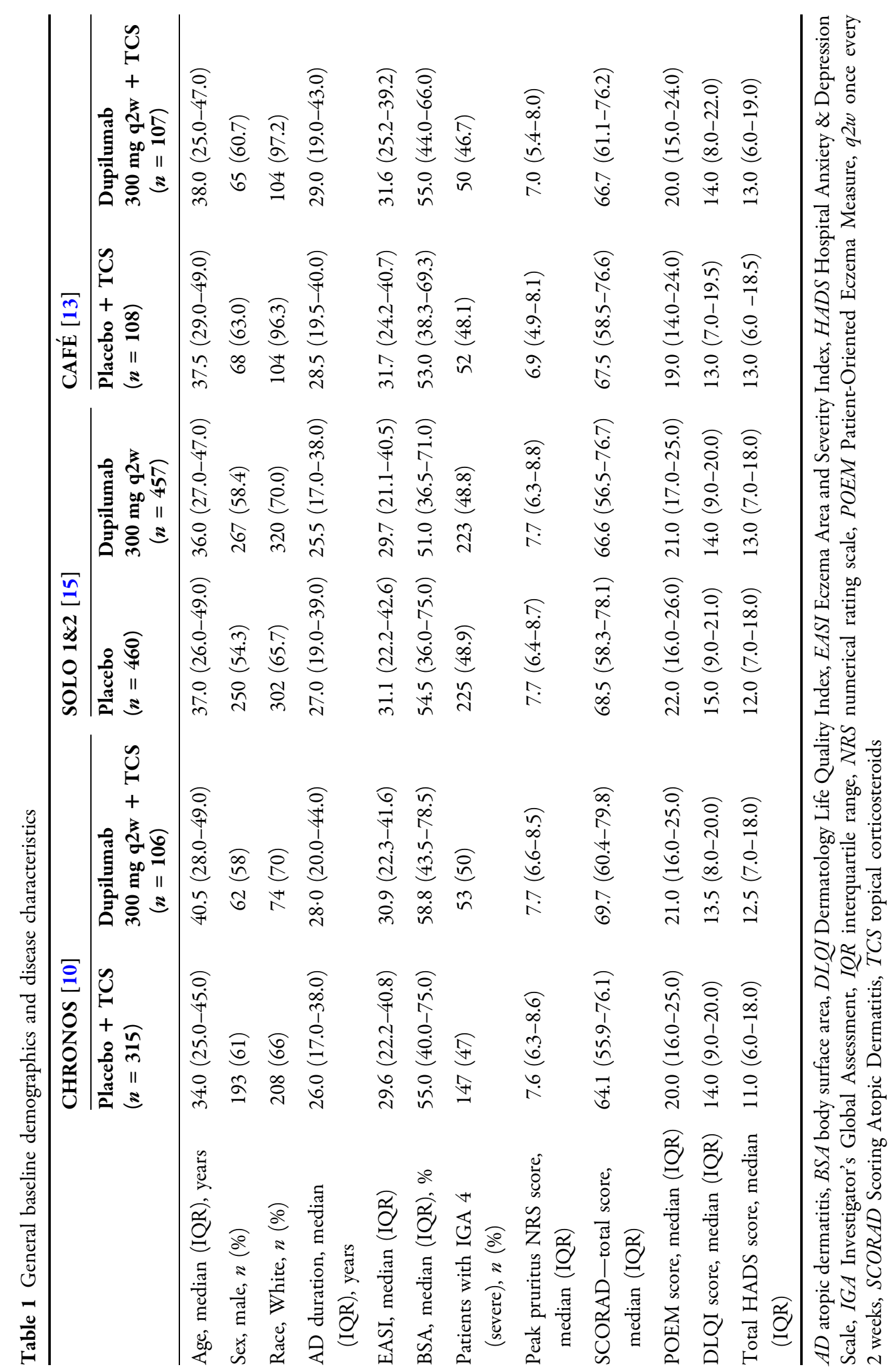




\section{Patient Global Assessment of Disease Status (PGADS)}

In all four studies, a significantly higher proportion of dupilumab-treated patients reported a "Good"/"Very Good"/"Excellent" disease status at end of study versus control (16 weeks, SOLO $1 \& 2$ : $59.5 \%$ vs. $24.6 \%$; CAFÉ: $84.1 \%$ vs. $45.4 \%$; 52 weeks, CHRONOS: $69.8 \%$ vs. $25.1 \%$ all $P<0.0001$ ) (Fig. 1). A significant difference in patients' perceptions of $\mathrm{AD}$ improvement for the dupilumab versus control group was seen by week 2 in all studies that continued increasing until week 6 (all studies, $P<0.0001$ ) and was maintained until the end of all four studies (Fig. 2).

\section{Patient Global Assessment of Treatment Effect (PGATE)}

Significantly more dupilumab-treated patients reported treatment effect as "Good"/"Very Good"|"Excellent" at the end of all four studies versus control (16 weeks, SOLO $1 \& 2: 65.0 \%$ vs. $21.1 \%$; CAFÉ: $85.0 \%$ vs. $36.1 \%$; 52 weeks, CHRONOS: $72.6 \%$ vs. $24.8 \%$; all $P<0.0001$ )

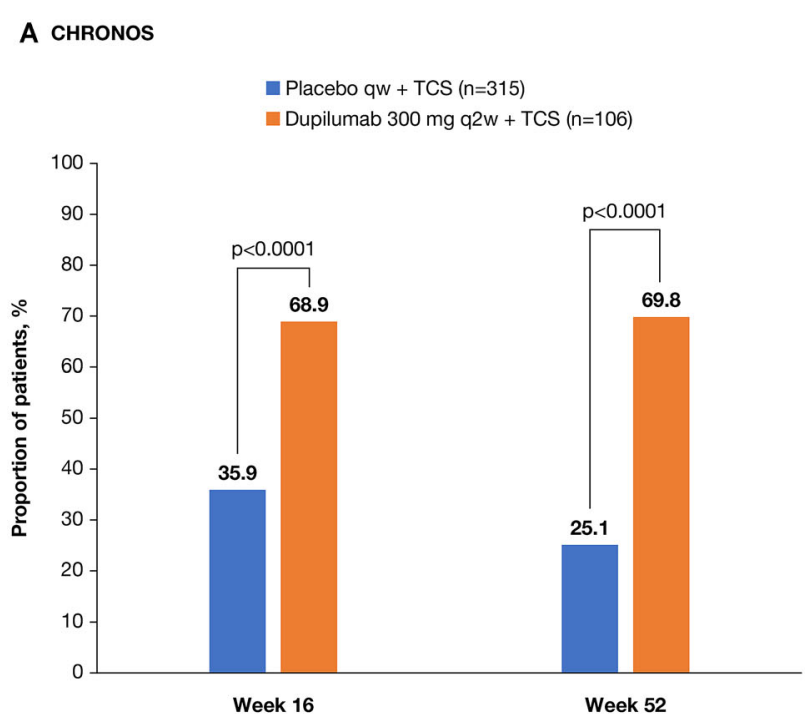

Fig. 1 Proportion of patients achieving good/very good/ excellent response in PGADS at the end of study (week 16 and week $52^{\mathrm{a}}$ ), FAS ${ }^{\mathrm{b}}$. ${ }^{\mathrm{a}}$ Week 16 for CHRONOS, SOLO 1\&2, and CAFÉ; week 52 for CHRONOS only;
(Fig. 3). As with PGADS, a difference in patients' perceptions of treatment efficacy for dupilumab versus control was apparent by week 2 in all studies (Fig. 2). In general, this treatment difference continued to increase until week 6 (all studies, $P<0.0001)$ and was maintained until end of treatment (all studies, $P<0.0001$ ) (Fig. 2).

\section{DISCUSSION}

In this combined analysis of patient-reported disease improvement (PGADS) and patient-reported treatment efficacy (PGATE) data from four randomized, double-blind, placebo-controlled, pivotal phase 3 trials [9, 10, 13, 15], adult patients with $\mathrm{AD}$ receiving dupilumab with/without TCS perceived greater improvements in disease status and treatment effect versus patients treated with control. Dupilumab-treated patients across all four studies perceived improvement of their $\mathrm{AD}$ as early as week 2 and this improvement was maintained through week 16 (SOLO 1\&2, CAFÉ) and week 52 (CHRONOS). These results reflect those of previous publications demonstrating the

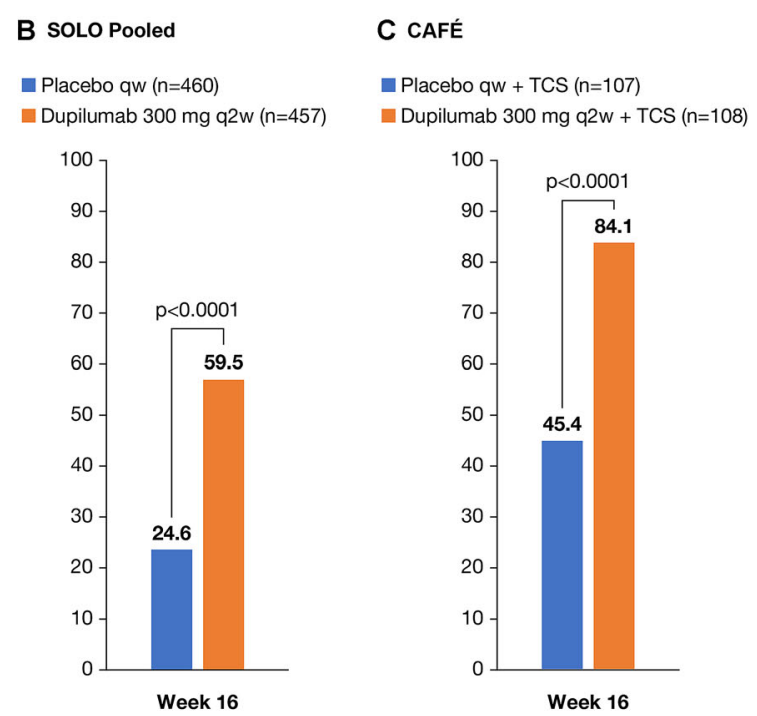

${ }^{\mathrm{b}}$ Patient considered non-responder after rescue medication use. FAS full analysis sample, PGADS Patient Global Assessment of Disease Status, $\mathrm{q} 2 \mathrm{w}$ once every 2 weeks, TCS topical corticosteroids 

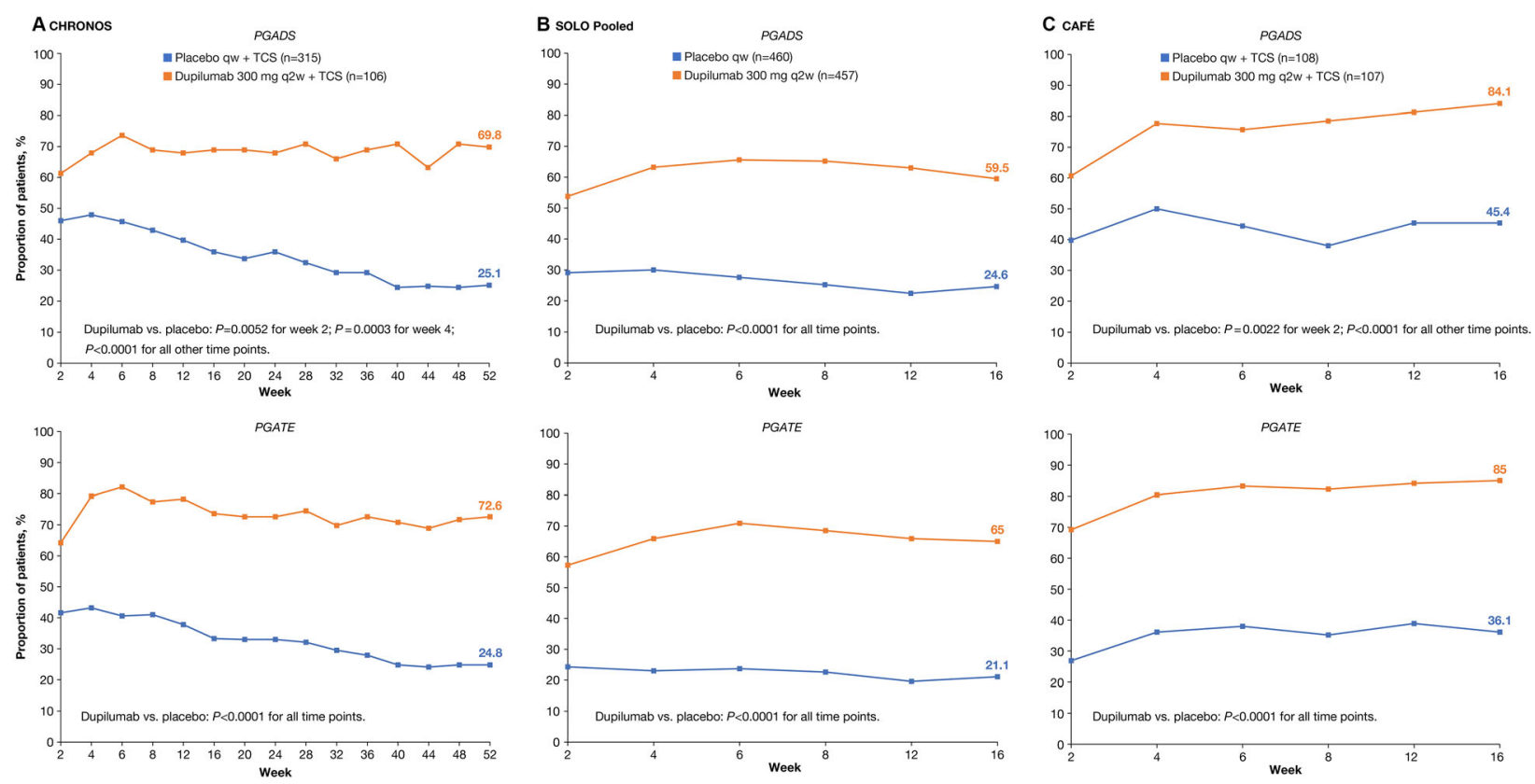

Fig. 2 Percentage of patients achieving good/very good/ excellent response in PGADS and PGATE overtime, FAS $^{\mathrm{a}, \mathrm{b}}$. ${ }^{\mathrm{a}}$ Week 16 for CHRONOS, SOLO 1\&2, and CAFÉ; week 52 for CHRONOS only; ${ }^{\mathrm{b}}$ Patient considered non-responder after rescue medication use. FAS full analysis sample, PGADS Patient Global Assessment of Disease Status, PGATE Patient Global Assessment of Treatment Effect, q2w once every 2 weeks, TCS topical corticosteroids

\section{A CHRONOS}

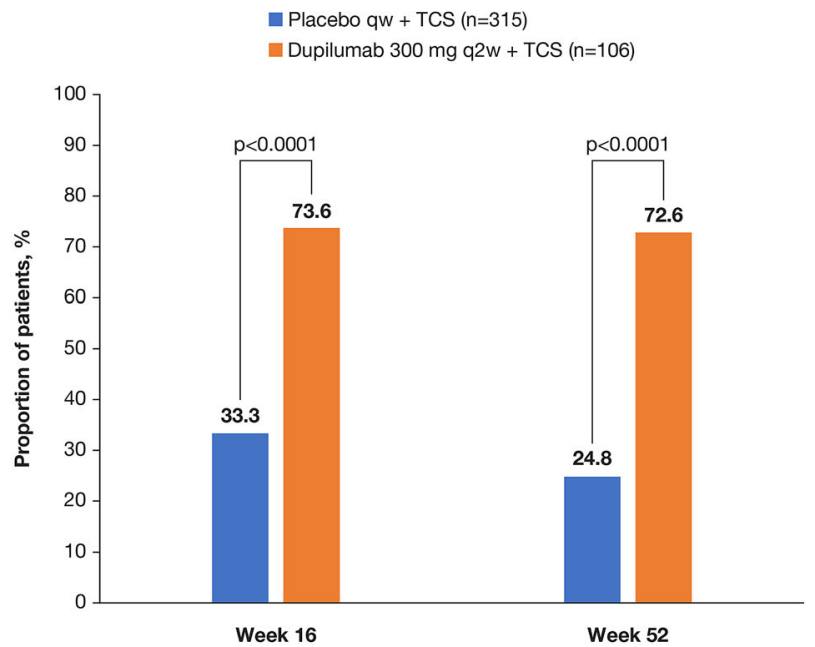

Fig. 3 Proportion of patients achieving good/very good/ excellent response in PGATE at the end of study (week 16 and week $52^{\mathrm{a}}$ ), FAS ${ }^{\mathrm{b}}$. ${ }^{\mathrm{a}}$ Week 16 for CHRONOS, SOLO 1\&2, and CAFÉ; week 52 for CHRONOS only; ${ }^{\mathrm{b}}$ Patient considered non-responder after rescue medication

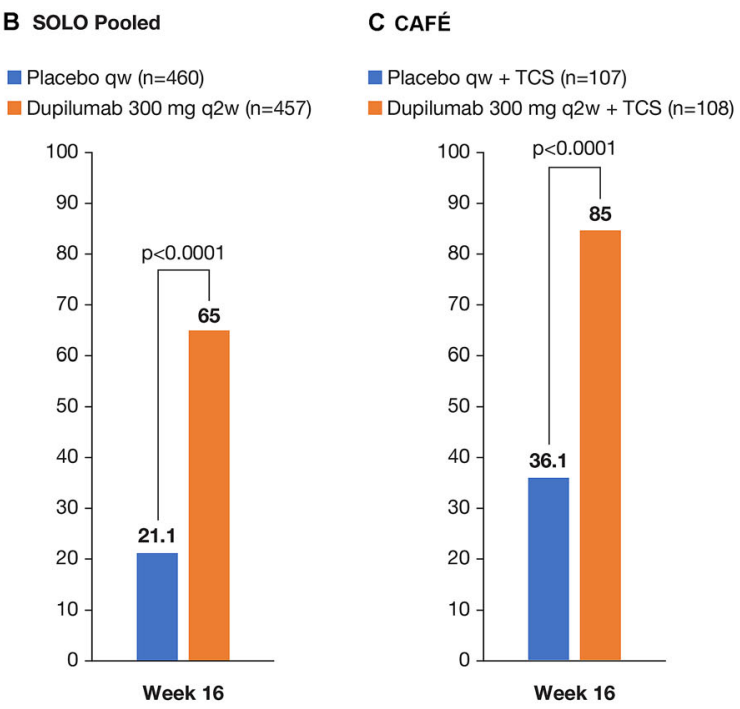

use. FAS full analysis sample, PGATE Patient Global Assessment of Treatment Effect, q2w once every 2 weeks, TCS topical corticosteroids 
rapid and sustained effect of dupilumab using physician-assessed outcomes measures, as well as patient-reported peak pruritus and a range of holistic QoL measures capturing overall patient well-being (Dermatology Life Quality Index, DLQI; Hospital Anxiety \& Depression Scale, HADS; Patient-Oriented Eczema Measure, POEM; and the 5-dimension 3-level EuroQol, EQ-5D) [7-15]. More importantly, the high percentage of patients reporting good perception of treatment effect (around 80\% across studies) is similar to the percentage of patients who persist on treatment with dupilumab in real-world studies with up to 2 years of followup $[22,23]$. In a retrospective cohort study of 1963 adult patients in the USA who received dupilumab treatment, the persistence (95\% confidence interval [CI]) at 6 and 12 months was $91.9 \% \quad(90.7-93.2 \%)$ and $77.3 \%$ (75.0-79.7\%), respectively [23]. In the BioDay registry, which included 402 adult patients receiving dupilumab across multiple centers in the Netherlands, the overall drug survival rates for dupilumab were $91 \%$ and $88 \%$ after 1 and 2 years, respectively.

In a real-world cohort including 109 adults and children treated with dupilumab, the proportion $(95 \% \mathrm{CI})$ of patients with persistence of remaining on dupilumab treatment at 12,24 , and 36 months was $96.5 \%$ (92.7-99.9\%), 88.1\% (83.9-98.5\%), and 78.9\% (64.4-99.9\%), respectively [23]. Given that traditional clinical outcome measures used to evaluate $\mathrm{AD}$ [e.g., IGA and Eczema Area and Severity Index (EASI)] may not fully capture patient-reported burden $[3,20,21]$, this analysis addresses an important gap in knowledge. These results provide insight into patient impressions of the efficacy of dupilumab for the treatment of moderate-tosevere $\mathrm{AD}$ and are a component of the holistic and meaningful improvement seen with dupilumab.

A potential limitation of this analysis is that it is based on data from clinical trials which may not be reflective of dupilumab use in a realworld setting. However, the results of this analysis are supported by recently published real-world data showing high persistence levels and low treatment discontinuation in patients treated with dupilumab for up to 3 years
$[22,24,25]$. A further potential limitation of this analysis is that both measures used to assess disease status and treatment effect have not been formally validated.

\section{CONCLUSION}

Adult patients with moderate-to-severe $\mathrm{AD}$ treated with dupilumab with/without TCS perceived treatment as highly effective and as improving overall well-being, as early as 2 weeks and throughout treatment periods up to a year.

\section{ACKNOWLEDGEMENTS}

Funding. Research sponsored by Sanofi and Regeneron Pharmaceuticals, Inc. The Dermatology and Therapy rapid service fee was funded by Sanofi and Regeneron Pharmaceuticals Inc.

Medical Writing and Editorial Assistance. Medical writing and editorial assistance was provided by Yunyu Huang of Excerpta Medica, funded by Sanofi Genzyme and Regeneron Pharmaceuticals, Inc. according to the Good Publication Practice guideline [26].

Authorship. All named authors meet the International Committee of Medical Journal Editors (ICMJE) criteria for authorship for this article, take responsibility for the integrity of the work as a whole, and have given their approval for this version to be published.

Author Contributions. ABR, DD, DS contributed to study concept and design, analysis, and interpretation, provided critical feedback on the manuscript, approved the final manuscript for submission, and were accountable for the accuracy and integrity of the manuscript. ZC conducted the statistical analyses on the data. JFM, MBW, CHH, ESB, KE interpreted the data, provided critical feedback on the manuscript, approved the final manuscript for submission, and were accountable for the accuracy and integrity of the manuscript. 
Disclosures. Marjolein de Bruin-Weller: Regeneron Pharmaceuticals, Inc., Sanofi Genzyme-Principal Investigator, advisory board member, consultant; AbbVie, LEO Pharma, Pfizer-Principal Investigator, advisory board member; Eli Lilly, Galderma, Janssen, UCBadvisory board member. Joseph F. Merola: Regeneron Pharmaceuticals, Inc., Sanofi Genzyme-Principal Investigator, advisory board member, consultant. Chih-Ho Hong: Arcutis, Centocor, Cutanea, MedImmune-investigator; Sanofi-Aventis-consultant; Boehringer Ingelheim, GlaxoSmithKline-investigator, consultant; Cipher-speaker; AbbVie, Amgen, Bausch Health, BMS, Dermavant, Eli Lilly, Galderma, Janssen, LEO Pharma, Novartis, Regeneron Pharmaceuticals, Inc., Roche, Sun Pharma, UCB-speaker, investigator, consultant. Esther Serra Baldrich: Regeneron Pharmaceuticals, Inc., Sanofi Genzyme-Principal Investigator, advisory board member, consultant. Karel Ettler: Regeneron Pharmaceuticals, Inc., Sanofi Genzyme-Principal Investigator, advisory board member, consultant. Dimittri Delevry, Zhen Chen: Regeneron Pharmaceuticals, Inc.employees and shareholders. Ana B. Rossi, Debra Sierka: Sanofi Genzyme-employee, may hold stock and/or stock options in the company.

Compliance with Ethics Guidelines. All trials were approved by respective institutional review boards and conducted in accordance with the ethical principles outlined in the Declaration of Helsinki, the International Conference on Harmonisation Good Clinical Practice guidelines, and applicable regulatory requirements. All patients or carers provided written informed consent before participating in the trial.

Data Availability. The datasets generated during and/or analyzed during the current study are available from the corresponding author on reasonable request.

Open Access. This article is licensed under a Creative Commons Attribution-NonCommercial 4.0 International License, which permits any non-commercial use, sharing, adaptation, distribution and reproduction in any medium or format, as long as you give appropriate credit to the original author(s) and the source, provide a link to the Creative Commons licence, and indicate if changes were made. The images or other third party material in this article are included in the article's Creative Commons licence, unless indicated otherwise in a credit line to the material. If material is not included in the article's Creative Commons licence and your intended use is not permitted by statutory regulation or exceeds the permitted use, you will need to obtain permission directly from the copyright holder. To view a copy of this licence, visit http://creative commons.org/licenses/by-nc/4.0/.

\section{REFERENCES}

1. Brandt EB, Sivaprasad U. Th2 cytokines and atopic dermatitis. J Clin Cell Immunol. 2011;2(3):110.

2. Barbarot S, Auziere S, Gadkari A, et al. Epidemiology of atopic dermatitis in adults: results from an international survey. Allergy. 2018;73(6):1284-93.

3. Simpson EL, Bieber T, Eckert L, et al. Patient burden of moderate to severe atopic dermatitis (AD): insights from a phase $2 \mathrm{~b}$ clinical trial of dupilumab in adults. J Am Acad Dermatol. 2016;74(3):491-8.

4. Silverberg JI, Garg NK, Paller AS, Fishbein AB, Zee PC. Sleep disturbances in adults with eczema are associated with impaired overall health: a US population-based study. J Investig Dermatol. 2015; 135(1):56-66.

5. Sibbald C, Drucker AM. Patient burden of atopic dermatitis. Dermatol Clin. 2017;35(3):303-16.

6. Gandhi NA, Bennett BL, Graham NM, Pirozzi G, Stahl N, Yancopoulos GD. Targeting key proximal drivers of type 2 inflammation in disease. Nat Rev Drug Discov. 2016;15(1):35-50.

7. Beck LA, Thaci D, Hamilton JD, et al. Dupilumab treatment in adults with moderate-to-severe atopic dermatitis. N Engl J Med. 2014;371(2):130-9.

8. Thaci D, Simpson EL, Beck LA, et al. Efficacy and safety of dupilumab in adults with moderate-tosevere atopic dermatitis inadequately controlled by topical treatments: a randomised, placebo-controlled, dose-ranging phase $2 \mathrm{~b}$ trial. Lancet. 2016;387(10013):40-52. 
9. Simpson EL, Bieber T, Guttman-Yassky E, et al. Two phase 3 trials of dupilumab versus placebo in atopic dermatitis. N Engl J Med. 2016;375(24):2335-48.

10. Blauvelt A, de Bruin-Weller M, Gooderham M, et al. Long-term management of moderate-to-severe atopic dermatitis with dupilumab and concomitant topical corticosteroids (LIBERTY AD CHRONOS): a 1-year, randomised, double-blinded, placebo-controlled, phase 3 trial. Lancet. 2017;389(10086): 2287-303.

11. Simpson EL. Dupilumab improves general healthrelated quality-of-life in patients with moderate-tosevere atopic dermatitis: pooled results from two randomized, controlled phase 3 clinical trials. Dermatol Ther (Heidelb). 2017;7(2):243-8.

12. Simpson EL, Gadkari A, Worm M, et al. Dupilumab therapy provides clinically meaningful improvement in patient-reported outcomes (PROs): a phase IIb, randomized, placebo-controlled, clinical trial in adult patients with moderate to severe atopic dermatitis (AD). J Am Acad Dermatol. 2016;75(3): 506-15.

13. de Bruin-Weller $\mathrm{M}$, Thaci $\mathrm{D}$, Smith $\mathrm{CH}$, et al. Dupilumab with concomitant topical corticosteroid treatment in adults with atopic dermatitis with an inadequate response or intolerance to ciclosporin $\mathrm{A}$ or when this treatment is medically inadvisable: a placebo-controlled, randomized phase III clinical trial (LIBERTY AD CAFE). Br J Dermatol. 2018; 178(5):1083-101.

14. Deleuran M, Thaci D, Beck LA, et al. Dupilumab shows long-term safety and efficacy in patients with moderate to severe atopic dermatitis enrolled in a phase 3 open-label extension study. J Am Acad Dermatol. 2020;82(2):377-88.

15. Thaci D, Simpson EL, Deleuran M, et al. Efficacy and safety of dupilumab monotherapy in adults with moderate-to-severe atopic dermatitis: a pooled analysis of two phase 3 randomized trials (LIBERTY AD SOLO 1 and LIBERTY AD SOLO 2). J Dermatol Sci. 2019;94(2):266-75.

16. DUPIXENT ${ }^{\circledR}$ (dupilumab) injection, for subcutaneous use [prescribing information]. Regeneron Pharmaceuticals, Inc, Tarrytown, NY2020. 2017. https://www.accessdata.fda.gov/drugsatfda_docs/ label/2020/761055s020lbl.pdf. Accessed 1 Feb 2021.

17. European Medicines Agency. Dupixent (dupilumab). Accessed 1 Feb 2021; https://www.ema. europa.eu/en/medicines/human/EPAR/dupixent\# authorisation-details-section. Accessed 1 Feb 2021.
18. EMA. European Medicines Agency: DUPIXENT ${ }^{\circledR}$ (dupilumab). Summary of product characteristics. 2021. https://ec.europa.eu/health/documents/com munity-register/2019/20190801145601/anx_14560 1_en.pdf. Accessed Mar 2021.

19. Bruin-Weller M, Biedermann T, Bissonnette R, et al. Treat-to-target in atopic dermatitis: an international consensus on a set of core decision points for systemic therapies. Acta Dermato Venereol. 2021; 101(2):00402.

20. Wei W, Anderson P, Gadkari A, et al. Discordance between physician- and patient-reported disease severity in adults with atopic dermatitis: a US crosssectional survey. Am J Clin Dermatol. 2017;18(6): 825-35.

21. Cork MJ, Eckert L, Simpson EL, et al. Dupilumab improves patient-reported symptoms of atopic dermatitis, symptoms of anxiety and depression, and health-related quality of life in moderate-tosevere atopic dermatitis: analysis of pooled data from the randomized trials SOLO 1 and SOLO 2. J Dermatol Treat. 2020;31(6):606-14.

22. Spekhorst LS, Ariëns LFM, Schaft J, et al. Two-year drug survival of dupilumab in a large cohort of difficult-to-treat adult atopic dermatitis patients compared to cyclosporine A and methotrexate: results from the BioDay registry. Allergy. 2020; 75(9):2376-9.

23. Silverberg JI, Guttman-Yassky E, Gadkari A, et al. Real-world persistence with dupilumab among adults with atopic dermatitis. Ann Allergy Asthma Immunol. 2021;126(1):40-5.

24. Ariens LF, van der Schaft J, Spekhorst LS, et al. Dupilumab shows long-term effectiveness in a large cohort of treatment-refractory atopic dermatitis patients in daily practice: 52 -weeks results from the Dutch BioDay registry. J Am Acad Dermatol. 2021;84(4):1000-9.

25. Halling AS, Loft N, Silverberg JI, Guttman-Yassky E, Thyssen JP. Real-world evidence of dupilumab efficacy and risk of adverse events: a systematic review and meta-analysis. J Am Acad Dermatol. 2021; 84(1):139-47.

26. Battisti WP, Wager E, Baltzer L, et al. Good publication practice for communicating company-sponsored medical research: GPP3. Ann Intern Med. 2015;163(6):461-4. 